\title{
GERAÇÃO DE ENERGIA FOTOVOLTAICA: ANÁLISE COMPARATIVA ENTRE REDENÇÃO NO BRASIL E MAPUTO EM MOÇAMBIQUE
}

\author{
Túlio Pinheiro Moura ${ }^{1}$, Juan Carlos Alvarado Alcócer ${ }^{2}$, Olienaide Ribeiro de \\ Oliveira Pinto ${ }^{3}$, Luís Tomás Domingos ${ }^{4}$.
}

${ }^{1}$ Mestrando no Mestrado Acadêmico em Sociobiodiversidade e Tecnologias Sustentáveis (MASTS) da Universidade da Integração Internacional da Lusofonia Afro-Brasileira (UNILAB).

${ }^{2}$ Professor Doutor da Universidade da Integração Internacional da Lusofonia AfroBrasileira (UNILAB), Redenção/CE, Brasil.

${ }^{3}$ Doutora, Bolsista do Programa Nacional de Pós-Doutorados (PNPD) /CAPES da Universidade da Integração Internacional da Lusofonia Afro-Brasileira (UNILAB), Redenção/CE, Brasil. Email: agron.olienaide@gmail.com

${ }^{4}$ Professor Doutor da Universidade da Integração Internacional da Lusofonia AfroBrasileira (UNILAB), Redenção/CE, Brasil.

\section{Recebido em: 06/04/2019 - Aprovado em: 10/06/2019 - Publicado em: 30/06/2019 DOI: 10.18677/EnciBio_2019A179}

\begin{abstract}
A energia solar fotovoltaica é uma fonte limpa e alternativa de geração de energia elétrica. É uma opção na atual matriz energética brasileira e também moçambicana, mostrando-se uma tendência de mercado que pode substituir as fontes fósseis de energia. Assim, este artigo de revisão de literatura teve como objetivo estudar o comparativo da energia gerada a partir de sistemas fotovoltaicos em Redenção no Ceará e Maputo em Moçambique. Considerou-se uma unidade consumidora (UC) localizada na Província de Maputo e outra UC localizada na Cidade de Redenção. Padronizou-se o mesmo consumo médio mensal de energia elétrica de 794 kWh para as duas cidades. A partir dos dados de irradiação das cidades, foi estimada a geração para um módulo fotovoltaico para verificar qual cidade apresentou maior geração de energia solar. Determinou-se a potência instalada para cada cidade, e verificou-se a mais viável para a instalação do sistema fotovoltaico. Com resultados, a região de Maputo apresentou maior tendência de geração de energia em comparação à cidade de Redenção, devido possuir maiores índices de irradiação. Em Maputo, o sistema fotovoltaico foi $4,74 \mathrm{kWp}$ que instalado pode atender 0 consumo médio mensal. Observou-se que, a potência instalada para a UC de Maputo é menor do que a UC de Redenção, isso é devido Maputo apresentar maiores índices de irradiação ao logo do ano em comparação aos dados de Redenção. Conclui-se que, as duas cidades apresentaram dados favoráveis para geração de energia fotovoltaica.
\end{abstract}

RESUMO

PALAVRAS-CHAVE: Energia Solar Fotovoltaica. Irradiação. Projetos Fotovoltaicos. 


\title{
PHOTOVOLTAIC ENERGY PRODUCTION: COMPARATIVE ANALYSIS BETWEEN REDENÇÃO / BRASIL AND MAPUTO / MOÇAMBIQUE
}

\begin{abstract}
Photovoltaic solar energy is a clean and alternative source of electric power generation. It is an option in the current Brazilian and also Mozambican energy matrix, showing a market trend that can replace fossil energy sources. Thus, the objective of the work was to study the comparative of the energy generated from photovoltaic systems in Redenção in Ceará and Maputo in Mozambique. It was considered a consumer unit (UC) located in Maputo Province and another UC located in the City of Redenção. The average monthly electricity consumption of 794 $\mathrm{kWh}$ was standardized for both cities. From the irradiance data of the cities, it was estimated the generation for a photovoltaic module to verify which city presented the highest generation of solar energy. The installed power was determined for each city, and it was verified the most feasible for the installation of the photovoltaic system. With results, the region of Maputo presented a greater trend of energy generation compared to the city of Redenção, due to its higher irradiation rates. In Maputo, the photovoltaic system with about $4.74 \mathrm{kWp}$ installed can meet the average monthly consumption. It was observed that, the installed power for the Maputo CU is lower than the UC of Redenção; this is due to Maputo presenting higher irradiance rates to the year's logo in comparison to the Redenção data. It is concluded that the two cities presented favorable data for the generation of photovoltaic energy.
\end{abstract}

KEYWORDS: Photovoltaic Solar Energy. Irradiation. Photovoltaic Projects.

\section{INTRODUÇÃO}

A crescente inquietação com a preservação do meio ambiente e a demanda pela diversificação da matriz energética, associado com o aumento na demanda por energia e desenvolvimento da indústria, impulsionou a geração de energia elétrica no mundo a partir de fontes renováveis, como a fonte solar (NASCIMENTO, 2017).

A implantação de sistemas fotovoltaicos conectados à rede elétrica simboliza uma forma de reduzir os custos de energia elétrica de vários setores: tanto para projetos residenciais e indústrias como também para centros comerciais e no setor agrícola. A energia elétrica é um bem de consumo diretamente relacionado ao desenvolvimento econômico e social de qualquer país, por essa razão a mesma apresenta uma tendência de demanda crescente ao passar dos anos, com leves oscilações em períodos de crise energética e/ou econômica (BRASIL, 2014).

Logo, a incessante busca pela expansão energética traz a necessidade de diversificação da matriz de produção de energia elétrica brasileira ou moçambicana, o que intensificou o uso das fontes de energias renováveis como complemento às fontes convencionais tanto do Brasil, quanto em Moçambique BRASIL, 2014).

Nesse contexto, vem abrindo cada vez mais espaço para Geração Distribuída (GD) de energia elétrica no Brasil e Moçambique, que é relativamente recente quando comparada a países europeus. Geração Distribuída (GD) refere-se à geração de energia que se caracteriza por estar próxima do consumidor, portanto economiza no sistema de transmissão e nas perdas do sistema em geral (INEE, 2018). Essa geração ocorre independe de potência, tecnologia e fonte de energia. Portanto, citam-se como exemplos de geração distribuída os co-geradores, painéis fotovoltaicos, geradores de emergência e pequenas centrais elétricas (BORGES, 2017).

No Brasil as Gerações Distribuídas (GDs) de pequeno porte foram regulamentas através das resoluções $n^{\circ} 482 / 2012$ (ANEEL, 2012) e $n^{\circ} 687 / 2015$ da 
Agência Nacional de Energia Elétrica (ANEEL, 2015). Em Moçambique a energia solar é regulada sob responsabilidade da Autoridade Reguladora de Energia (SANTOS et al., 2013). Essas GDs de pequeno porte abriram então espaço para um forte crescimento da fonte solar na geração elétrica brasileira, que em maio de 2017 representou $70 \%$ do total da potência instalada de geração distribuída no país, seguido pela eólica com 9\% (ANEEL, 2017).

O Brasil possui um grande potencial para gerar eletricidade através da conversão fotovoltaica, todavia ainda não amplamente aproveitado. A Alemanha, por exemplo, está entre os líderes no uso de energia solar fotovoltaica com uma insolação 40\% menor do que na região menos ensolarada do Brasil (TAB ENERGIA 2019). A energia solar no Brasil, diariamente incide entre $4.500 \mathrm{Wh} / \mathrm{m}^{2}$ a 6.300 $\mathrm{Wh} / \mathrm{m}^{2}$. Isso demonstra que as condições brasileiras são favoráveis ao aproveitamento dessa fonte na complementação de sua matriz elétrica. Estima-se que a capacidade instalada de geração solar chegue a $8.300 \mathrm{MW}$ em 2024, sendo 1.300 MW de geração distribuída (BRASIL, 2017).

Em termos de recursos renováveis disponíveis, o Atlas de Energias Renováveis de Moçambique em 2013, indica que o país dispõe de um espectro teórico diversificado que perfaz um total de $23.026 \mathrm{GW}$, sendo a energia solar a fonte de energia mais abundante (23.000 GW), seguida da hídrica (19 GW), eólica (5 GW), biomassa (2 GW) e a geotermia (0,1 GW) (ALER, 2018). Tendo em vista a diversidade de fontes mencionadas, Moçambique apresenta condições favoráveis para satisfazer a procura interna e para exportar para os países da região da África Austral e para outros mercados internacionais.

O uso de energia solar no Brasil e em Moçambique se apresenta como uma alternativa energética. Um dos fatores que tornam o uso da energia solar uma das melhores escolhas dentre as opções de geração de energia renováveis, é a fácil adaptação e instalação dos projetos, tanto para projetos residenciais e indústrias como também para centros comerciais e no setor agrícola (TAB ENERGIA 2019).

Diante deste cenário, é possível compreender o crescente interesse do mercado em projetos fotovoltaicos, tanto do lado da demanda como da oferta para os dois países. Nesta perspectiva, este artigo de revisão de literatura teve como objetivo contribuir para análise comparativa da produção de energia solar fotovoltaica entre a cidade de Redenção no Brasil e a província de Maputo em Moçambique, apresentando os procedimentos de dimensionamento de um sistema a ser conectado à rede elétrica em duas regiões distintas.

\section{Energia solar fotovoltaica para o Brasil e Moçambique}

A demanda por fontes de energia renováveis é um dos grandes desafios enfrentados pela humanidade nos últimos anos e vem tendo maior importância com ao aumento do efeito estufa. Uma das formas mais viáveis de amenizar esse problema é aproveitar a energia fornecida pelo sol, fonte limpa e gratuita de energia (DANTAS; POMPERMAYER, 2018).

$\mathrm{O}$ aproveitamento da fonte solar para gerar energia elétrica favorece diversos benefícios, tanto do ponto de vista elétrico, ambiental e socioeconômico. No enfoque elétrico, contribui para diversificação da matriz, aumento da segurança no fornecimento, redução de perdas e alívio de transformadores e alimentadores. No aspecto ambiental, ocorre a diminuição da emissão de gases do efeito estufa, da emissão de materiais particulados e do uso de água para geração de energia elétrica. Em relação aos benefícios socioeconômicos, a geração de energia solar 
fotovoltaica colabora com a geração de empregos locais, aumento da arrecadação e o aumento de investimentos (ABSOLAR, 2016).

O Brasil, no final de 2016, tinha $81 \mathrm{MWp}$ de energia solar fotovoltaica instalados, o que representou cerca de $0,05 \%$ da capacidade instalada total no país. Do total de $81 \mathrm{MWp}, 24 \mathrm{MWp}$ correspondiam à geração centralizada e $57 \mathrm{MWp}$ à Geração Distribuída (BRASIL, 2017). A baixa utilização da energia solar no Brasil chama mais a atenção quando se observam as condições favoráveis ao desenvolvimento da fonte no país, já que este possui altos níveis de insolação (NASCIMENTO, 2017). A introdução de sistemas distribuídos de energia elétrica no setor elétrico brasileiro tem ocorrido de modo gradual e imperiosa em resposta à ausência dos combustíveis fósseis e à redução da disponibilidade centralizada de amplos potenciais hidrelétricos no país (BORGES, 2017).

Nos últimos tempos, têm se verificado o crescimento técnico e econômico das tecnologias de geração distribuída, fruto do amplo apoio de países interessados em GD. Por trás desse interesse, pode-se destacar a necessidade de reduzir as emissões de gases de efeito estufa e desenvolver matrizes energéticas baseadas em fontes renováveis e, assim, reduzindo a dependência de combustíveis fósseis (EPE, 2014). No Brasil, pode se utilizar qualquer fonte renovável, além da cogeração qualificada, denominando-se microgeração distribuída a central geradora com potência instalada até 75 quilowatts $(\mathrm{kW})$ e minigeração distribuída aquela com potência acima de $75 \mathrm{~kW}$ e menor ou igual a $5 \mathrm{MW}$ (sendo $3 \mathrm{MW}$ para a fonte hídrica), conectadas na rede de distribuição por meio de instalações de unidades consumidoras (ANEEL, 2017).

A média anual de irradiação global no Brasil apresenta-se bem distribuída, com médias relativamente altas em todo o território. Os valores de irradiação solar global incidente em qualquer região do território brasileiro (1500-2.500) são superiores aos da maioria dos países europeus, como Alemanha $\left(900-1250 \mathrm{Wh} / \mathrm{m}^{2}\right)$, França (900-1650 Wh/m²) e Espanha (1200-1850 Wh/m²), locais onde projetos de aproveitamentos solares são amplamente disseminados (PEREIRA et al., 2006).

As projeções para 2024 é que o potencial fotovoltaico brasileiro continue sendo explorado, com considerável elevação da potência instalada (ANEEL, 2017), conforme a Figura 1. Percebe-se também, que as projeções são crescentes para as gerações distribuídas (GDs) de energia fotovoltaica tanto para os setores comerciais e como residenciais (EPE, 2017).

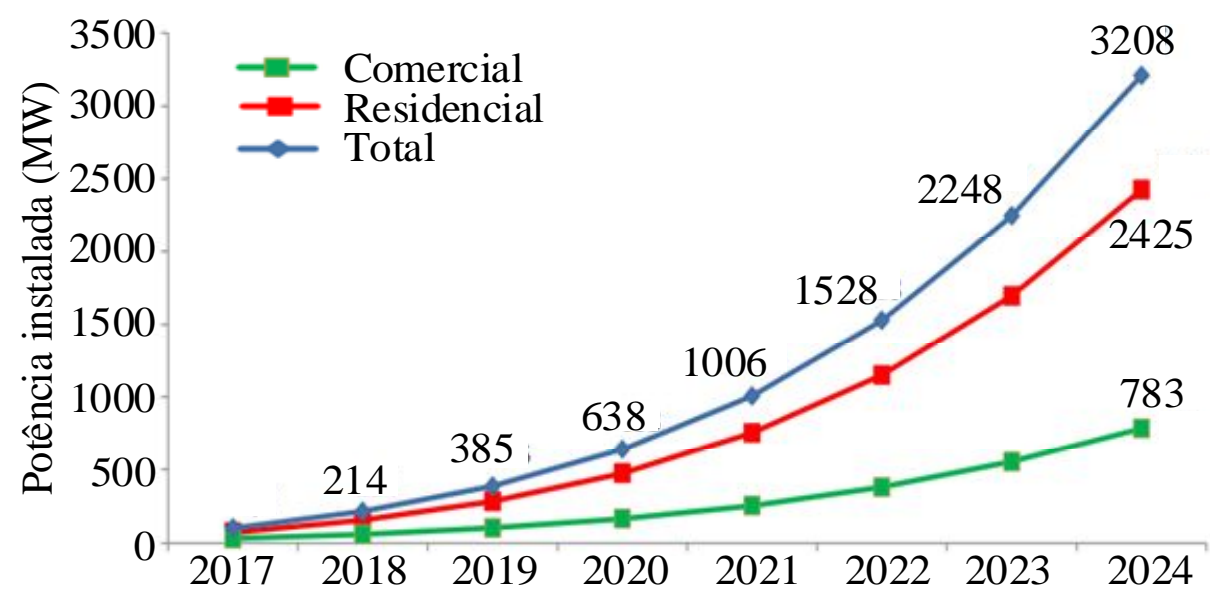

FIGURA 1. Projeções de GDs de fotovoltaicas.

Fonte: EPE (2017). 
Uma avaliação das condições energéticas de Moçambique, na África, através do ministério de energia, daquele governo, fornece dados importantes para conhecimento das condições de suas regiões desprovidas de energia elétrica (SANTOS et al., 2013).

Moçambique possui grandes recursos de energia de fontes renováveis, como solar, eólica e biomassa. Porém, apresenta pouco desenvolvido em relação a outros países no mundo, na mobilização destes recursos para produção de energia elétrica. Há diversas razões, como a pobreza, falta de financiamento, subsídios e conhecimento técnico e soluções de baixo custo. Entretanto, a falta de iniciativas políticas e investimento, são as principais (AIM, 2012).

O contexto em Moçambique se mostra, portanto, bastante conveniente para a geração de eletricidade com sistemas fotovoltaicos e, embora, a ênfase atual seja nos sistemas isolados, há a tendência mundial para geração utilizando sistemas fotovoltaicos conectados à rede. A aplicação desse tipo de sistema também pode chegar a Moçambique e o conhecimento do potencial de recurso solar e de geração fotovoltaica será fundamental para o crescimento da tecnologia no país (SANTOS et al., 2013).

Os projetos de geração de energia solar fotovoltaica dividem-se em projetos de geração centralizada, com usinas de maior porte, e de geração descentralizada, a chamada geração distribuída, localizada em casas, edifícios comerciais e públicos, condomínios e áreas rurais (NASCIMENTO, 2017). Apesar do grande número de incentivos para desenvolvimento da geração solar fotovoltaica e dos resultados obtidos nos últimos anos, ainda há muito que precisa ser feito para que a fonte solar se consolide na matriz energética brasileira e moçambicana.

Além do potencial brasileiro e moçambicano, outras vantagens são consideradas. De acordo com leva et al. (2004) o sistema possui fácil implantação; baixo custo de manutenção; alta modularidade; e, com o avanço tecnológico e incentivos governamentais pode favorecer a redução dos custos financeiros e competitividade no mercado.

\section{Procedimento de projeto de Sistema Fotovoltaico}

A demanda por projetos fotovoltaicos é crescente, visto suas vantagens socioambientais e financeiras. O sistema fotovoltaico básico considerado neste trabalho está representado na Figura 2. O sistema é formado por um arranjo de módulos fotovoltaicos (PV), um barramento CC de conexão dos mesmos, conversores CC/CC (para extração da máxima potência) e CC/CA, para adequação da tensão aos padrões da rede de conexão, um filtro passivo LC e a conexão com a concessionária.

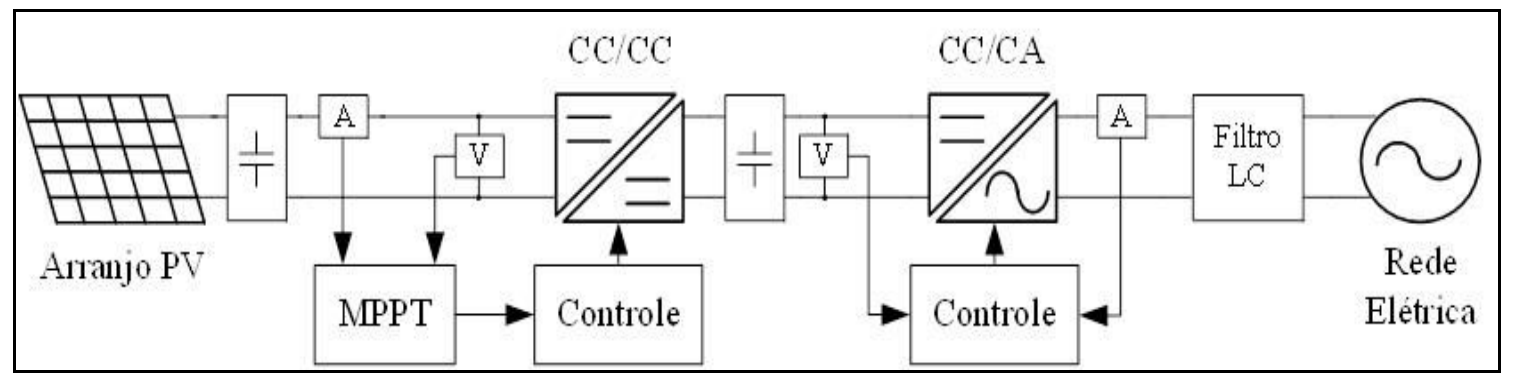

FIGURA 2. Sistema Fotovoltaico.

Fonte: Adaptado de Leva et al. (2004). 
Segundo Leva et al. (2004) os procedimentos de projeto para instalação de um sistema fotovoltaico são divididos em oito etapas, conforme descritos a seguir:

1. Caracterização geográfica do ambiente de instalação;

2. Estudo dos padrões de consumo do cliente;

3. Radiação incidente e orientação dos módulos PVs;

4. Dimensionamento do sistema PV;

5. Estudo de viabilidade (payback);

6. Solicitação de conexão com a concessionária local;

7. Instalação do Sistema PV;

8. Procedimento para conexão do sistema com a rede.

Há programas como o PVSystem ${ }^{\circledR}$ voltados para o dimensionamento de sistemas fotovoltaicos, porém, nesse trabalho detalhou-se as seguintes etapas abaixo desconsiderando a utilização de softwares específicos.

\section{Caracterização geográfica do ambiente de instalação}

A capacidade de geração de um sistema fotovoltaico está diretamente ligada as caraterísticas geográficas do local de instalação. O local escolhido para a instalação do sistema PV deve apresentar vantagens de insolação permanente durante todas as horas do dia para evitar o sombreamento nos módulos. No dimensionamento de um sistema fotovoltaico é necessário não somente avaliar o índice de radiação solar, mas também levar em consideração a temperatura do local, o que ainda não tem sido analisado na maioria dos projetos. O projetista deve ser criterioso na especificação dos equipamentos para garantir um projeto econômico e funcional (LEVA et al., 2004).

\section{Estudo dos padrões de consumo do cliente}

Um levantamento do consumo do cliente faz-se necessário para estimação da potência nominal do sistema. Para unidades consumidoras em baixa tensão, não é eficiente fazer o levantamento da potência instalada para se definir a carga instalada para o dimensionamento do sistema PV. Assim, faz-se o dimensionamento a partir do consumo mensal da Unidade Consumidora, por meio da fatura de energia, na qual trás o histórico de consumo dos ultimas 12 meses (em kWh) (BRASIL, 2015).

No Brasil, é cobrado um valor referente ao custo de disponibilidade da rede, dessa forma, mesmo que o sistema PV gere o suficiente para zerar a conta de energia, o titular da Unidade Consumidora deverá pagar a concessionária local o valor de disponibilidade (AYRES, 2019).

Este custo é definido na Resolução Normativa no 482/2012, onde as unidades consumidoras (UC) conectadas em baixa tensão (grupo B) deveram pagar um valor em reais equivalente a $30 \mathrm{kWh}$ (monofásico), $50 \mathrm{kWh}$ (bifásico) ou $100 \mathrm{kWh}$ (trifásico). O custo de disponibilidade também é cobrado para UC's conectados em alta tensão (grupo A), porém, o valor cobrado é correspondente à parcela da fatura relativa à demanda contratada (ANEEL, 2012).Em Moçambique não foi encontrada nenhuma informação sobre o custo de disponibilidade, no entanto neste artigo considerou-se o mesmo custo de disponibilidade com valores iguais aos do Brasil.

\section{Radiação incidente e orientação dos módulos PV}

Para o dimensionamento de qualquer sistema fotovoltaico, independente da aplicação, é fundamental ter o conhecimento da radiação solar incidente da região na qual o sistema será instalado (PEREIRA et al., 2006). 
No Brasil, o Atlas Solarimétrico elaborado pelo CRESESB, disponibiliza um banco de dados referentes as radiações incidentes em todo o período do ano. $O$ banco de dados da CRESESB disponibiliza a radiação média diária mensal para diferentes ângulos de incidência solar (PEREIRA et al., 2006).

Em Moçambique, a obtenção dos dados de irradiação foi pesquisada no Banco de Dados do Instituto Nacional de Meteorologia de Moçambique (INAM). O INAM realiza medições através de suas estações costeiras e do interior, abastecendo os órgãos governamentais e de pesquisa através de seu banco de dados (INAM, 2018).

O melhor ângulo de inclinação depende diretamente do local e tipo do sistema fotovoltaico onde será instalado. Independente do local é comum ser utilizado o mesmo ângulo da latitude do local para o ângulo de inclinação do módulo, não sendo indicada uma inclinação menor do que $10^{\circ}$, pois prejudicaria na manutenção dos módulos (CAMIOTO; GOMES, 2018).

Para sistemas conectados à rede elétrica (on-grid) no hemisfério sul, os módulos fotovoltaicos devem ser instalados com orientação, preferencialmente, em direção ao Norte Geográfico (SOUZA et al, 2016).

Para estimar a produção de energia gerada pelo sistema fotovoltaico, é útil ignorar os efeitos de variação da irradiação a cada instante e considerar uma totalidade de energia elétrica convertida em intervalos de horário. Assim, é conveniente expressar um valor acumulado de energia solar ao logo de um dia, conhecida como Horas de Sol Pleno (HSP). Esta grandeza mostra o número de horas equivalentes por dia em que a radiação solar permanece constante e igual a 1 $\mathrm{kW} / \mathrm{m}^{2}\left(1.000 \mathrm{~W} / \mathrm{m}^{2}\right)$, de modo que a energia resultante seja igual à energia disponibilizada pelo sol, acumulada ao logo de um dado dia (GTES, 2008).

De posse da irradiação incidente, é possível determinar as HSP diária equivalente à média anual da irradiação diária média mensal para o ângulo de $9^{\circ} \mathrm{N}$. De acordo com Gtes (2008) a equação (1) enuncia as horas de sol pleno diário que é dada por:

$$
H S P=\frac{\mathrm{I}_{\text {ine }}}{1 \mathrm{~kW} / \mathrm{m}^{2}}
$$

Onde:

- HSP: Horas de Sol Pleno [h /dia];

- Irradiação incidente $\left[\mathrm{kWh} / \mathrm{m}^{2}\right.$. dia].

\section{Dimensionamento do sistema PV}

Com base nos passos anteriores, define-se a potência e as especificações do módulo a ser adotado, assim como a quantidade necessária para atingir o valor nominal.

Segundo Cepel (2004), a potência instalada do sistema fotovoltaico para suprir totalmente ou parcialmente o consumo de energia mensal de uma edificação, é calculada a partir da equação (2):

$$
P_{F V}=\frac{\mathrm{C}_{\mathrm{m}}}{\text { TD.HSP. } 30}
$$

Onde:

- $P_{F V}$ : Potência Instalada [kWp]; 
- $\mathrm{C}_{\mathrm{m}}$ : Consumo médio mensal [kWh];

$T D$ : Taxa de desempenho [\%]

- HSP: Média mensal das horas de sol pleno [h].

A Taxa de Desempenho (TD) é um índice utilizado para avaliar a produção de energia de um sistema fotovoltaico levando em consideração as perdas envolvidas no sistema, por exemplo: sombreamento nos módulos, temperatura de operação dos módulos, perdas por carregamento e a eficiência do inversor (ZILLES et al., 2012).

Estudos indicam que as taxas de desempenho variam entre $60 \%$ a $90 \%$, sendo influenciado diretamente pelo aumento da eficiência dos inversores que, atualmente, têm alcançado eficiências em torno de $99 \%$. Um sistema fotovoltaico é avaliado como de boa qualidade, se a TD estiver acima de 80\% (PINHO; GOLDINO, 2014).

Segundo Zilles et al., (2012), a quantidade de módulos fotovoltaicos para atender a potência instalada do sistema é a relação da potência instalada sobre a potência de pico de um modelo de módulos fotovoltaico. A seguir é apresentada a equação (3) para determinar a quantidade de módulos.

$$
N_{\text {mod }}=\frac{\mathrm{P}_{\mathrm{FV}}}{\mathrm{P}_{\mathrm{MF}}}
$$

Onde:

- $N_{\text {modi }}$ : Número de Módulos;

- $\mathrm{P}_{\mathrm{FV}}$ : Potência Instalada [Wp];

- $\mathrm{P}_{\mathrm{MF}}$ : Potência de Pico de um módulo PV [Wp].

Segundo Nascimento (2017) é possível estimar a energia média mensal produzida por um módulo fotovoltaico a partir dos parâmetros de área e eficiência do módulo escolhido, e a média mensal anual das horas de sol pleno (HSP), conforme descrito na equação (4):

$$
E_{\text {mod }}=30 . A \cdot n_{\text {mod }} . H S P
$$

Onde:

- $E_{\text {mod }}$ : Energia média mensal gerada pelo módulo fotovoltaico [kWh];

- A: Àrea do módulo [m²];

- $n_{\text {mod }}$ : eficiência do Módulo [\%];

- HSP: Horas de Sol Pleno [h].

Para o dimensionamento do inversor, é necessário buscar inversores com maiores potências, capaz de comportar um maior número de módulos fotovoltaicos. Assim, a equação (5) determina a quantidade máxima de módulos para um inversor (CARNEIRO, 2009).

$$
N_{\text {mod } / \mathrm{inv}}=\frac{\mathrm{P}_{\mathrm{inv}}}{\mathrm{P}_{\mathrm{MF}}}
$$


Onde:

- $N_{\text {mod/inv }}$ : Número de módulos por inversores;

- $\mathrm{P}_{\text {inv }}$ : Potência nominal do inversor [W];

- $\mathrm{P}_{\mathrm{MF}}$ : Potência de Pico de um módulo PV [Wp].

A compatibilidade entre os módulos fotovoltaicos e o inversor é de extrema importância, visto que o principal fluxo de potência acontece entre a conexão de ambos, em que toda energia gerada pelos módulos é enviada à rede através do inversor. Caso não haja uma compatibilidade satisfatória, ocorrerá uma ineficiência na operação do sistema (ANEEL, 2016).

Assim, para um dimensionamento adequado do inversor, deve considerar o Fator de Dimensionamento do Inversor (FDI). Este conceito é de suma importância no dimensionado de sistemas fotovoltaicos por recomendar a capacidade do inversor em relação à potência de pico do módulo fotovoltaico, conforme apresentado na equação (6) (ABDALA, 2019).

$$
N_{i n v}=\frac{N_{m o d}}{N_{m o d / i n v}}
$$

Onde:

$N_{\text {inv }}$ : Número de inversores;

- $\mathrm{N}_{\text {mod }}$ : Número de Módulos PV;

- $N_{\text {mod/inv }}$ : Número de módulos por inversores.

Idealmente, toda a potência gerada pelos módulos fotovoltaicos deveria ser convertida pelo inversor e entregue à rede. Dessa forma, a relação entre a potência nominal do inversor e a do gerador fotovoltaico seria teoricamente a mesma, resultando em um FDI unitário e o processamento de energia no inversor seria ideal (PINHO; GOLDINO, 2014).

$\mathrm{Na}$ prática, as variações de irradiância ao longo do ano faz com que os arranjos fotovoltaicos não operem em condições nominais, pois seu MPPT também sofre variações, resultando em uma geração abaixo da potência nominal do gerador fotovoltaico. Neste caso, o inversor adotado pode ter uma potência nominal menor que a potência do gerador fotovoltaico sem que haja perda de eficiência do sistema, ocasionando em um subdimensionamento do inversor, de modo que o FDI será menor que a unidade. Para o caso da potência do inversor muito inferior a potência do arranjo, acarretará em um subdimensionamento excessivo, podendo ocorrer situações de pico de irradiância no qual o gerador fotovoltaico entregue uma potência de pico ao inversor superior a capacidade de processamento de energia total deste último, acarretando em perdas de potência (ZILLES et al., 2012).

Segundo Cepel (2004) o dimensionamento do inversor deve ser realizado de forma que o sistema não perca potência. Assim, a potência do inversor deve ser escolhida entre $70 \%$ a $120 \%$ da potência de pico do gerador fotovoltaico. Tendo uma faixa de FDI conforme equação (7):

$$
70 \%<F D I<120 \%
$$


De posse do número máximo de módulos fotovoltaicos por inversor, é necessário saber a quantidade de inversores para atender a potência instalada do sistema fotovoltaico. Assim, a equação (8) determina a quantidade mínima de inversores para suprir a potência instalada assim como o FDI (CARNEIRO, 2009).

$$
F D I=\frac{\mathrm{P}_{\mathrm{inv}}}{\mathrm{P}_{\mathrm{MF}}}
$$

Onde:

- FDI: Fator de dimensionamento do Inversor;

- $P_{\text {inv: }}$ : Potência nominal do inversor [W];

- $\mathrm{P}_{\mathrm{MF}}$ : Potência de Pico de um módulo PV [Wp].

\section{Comparativa da geração de energia fotovoltaica entre Maputo e Redenção}

Realizou-se um exemplo de projeto de Sistema Fotovoltaico comparando a geração de energia entre a cidade de Redenção e Maputo. Foi considerado o mesmo consumo médio mensal para as duas UCs de $794 \mathrm{kWh}$.

\section{Dados de Irradiação Solar}

Os dados de irradiação solar para Maputo e Redenção são apresentados na Tabela 1. Os dados de Maputo foram retirados a partir do banco de dados do Instituto Nacional de Meteorologia de Moçambique (INAM), conforme Santos et al., (2013), e os dados do Brasil foram retirados a partir do Banco de Dados da WEATHER SPARK.

TABELA 1. Irradiação solar diária média mensal para Maputo e Redenção.

\begin{tabular}{ccc}
\hline \multicolumn{3}{c}{ Irradiação solar diária média mensal $\left[\mathrm{kWh} / \mathrm{m}^{2}\right.$. dia $]$} \\
\hline Meses & $\begin{array}{c}\text { Maputo } \\
\text { Moçambique }\end{array}$ & $\begin{array}{c}\text { Redenção } \\
\text { Brasil }\end{array}$ \\
\hline Jan & 7,7 & 5,01 \\
Fev & 7,3 & 5,11 \\
Mar & 6,4 & 5,12 \\
Abr & 5,3 & 4,75 \\
Mai & 4,4 & 5,1 \\
Jun & 3,9 & 5,04 \\
Jul & 4,1 & 5,49 \\
Ago & 4,9 & 6,13 \\
Set & 5,8 & 6,29 \\
Out & 6,7 & 6 \\
Nov & 7,1 & 5,82 \\
Dez & 7,7 & 5,2 \\
\hline Média & $\mathbf{5 , 9 4}$ & $\mathbf{5 , 4 2}$ \\
\hline
\end{tabular}

Fonte: SANTOS et al., (2013) e WEATHER SPARK, (2019).

De acordo com o levantamento de dados ilustrados na Tabela 1, foi possível ilustrar nas Figuras 3 e 4, a curva de Irradiação Solar média diária mensal $\left(\mathrm{kWh} / \mathrm{m}^{2}\right.$ 
/dia) e a Energia Gerada a partir das HSP obtidas a partir da equação (4). Na Figura 3 , observa-se que os meses com maiores índices de irradiação incidente para a província de Maputo são de outubro a março. Em Maputo, a estação com precipitação é abafada e de céu quase sem nuvens; a estação seca é de ventos fortes e sem nuvens. Durante o ano inteiro, o clima é morno. Ao longo do ano, em geral a temperatura varia de $16{ }^{\circ} \mathrm{C}$ a $29^{\circ} \mathrm{C}$ e raramente é inferior a $14^{\circ} \mathrm{C}$ ou superior a $33^{\circ} \mathrm{C}($ INAM, 2018).

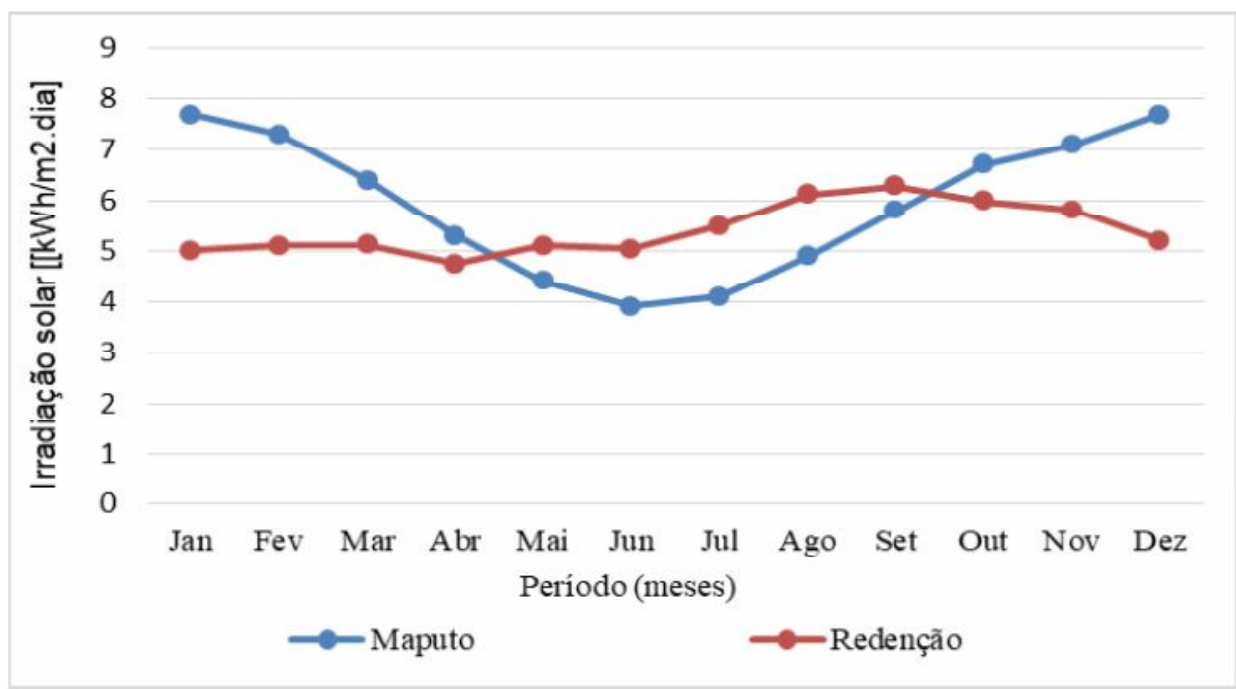

FIGURA 3. Dados de Irradiação solar diária média mensal.

Fonte: Autores (2019).

No caso de Redenção, o período com maior índice de irradiação é nos meses de maio a dezembro. Em Redenção, o verão é quente, árido e de ventos fortes; o inverno é longo, morno e com precipitação. Durante o ano inteiro, o tempo é opressivo e de céu parcialmente encoberto. Ao longo do ano, em geral a temperatura varia de $22^{\circ} \mathrm{C}$ a $35^{\circ} \mathrm{C}$ e raramente é inferior a $20^{\circ} \mathrm{C}$ ou superior a 36 ${ }^{\circ} \mathrm{C}$ (WEATHER SPARK, 2019).

Nota-se que na Figura 4, que a maior energia gerada é obtida a partir da cidade de Maputo, devido à mesma apresentar condições mais favoráveis de irradiação do que da cidade de Redenção. Estimou-se a energia média gerada em apenas um módulo da marca Bringing Green Power to Life para verificar qual sistema geraria mais energia. Assim, a estimativa de energia média gerada para Maputo é igual a $58 \mathrm{kWh}$ e para Redenção é de $53 \mathrm{kWh}$. Os dados do módulo fotovoltaico são apresentados na Tabela 2. 


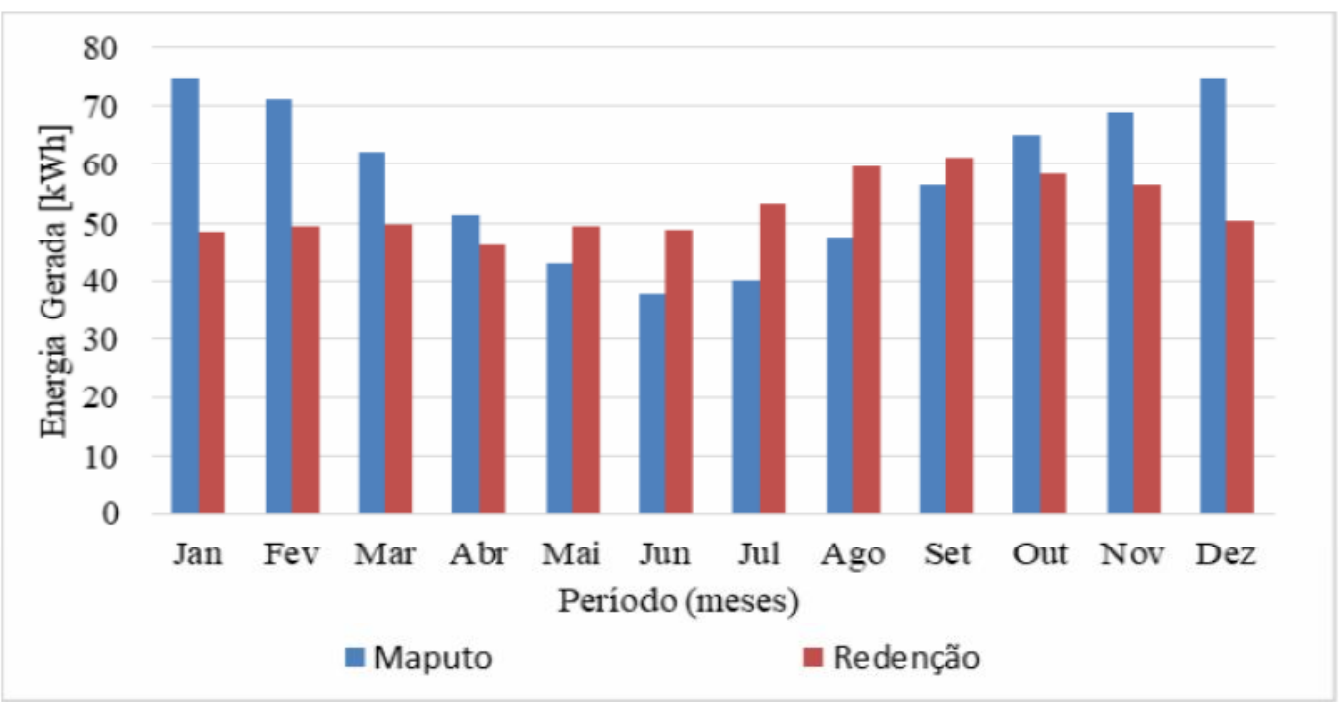

FIGURA 4. Energia Gerada (kWh).

Fonte: Autores (2019).

No quadro 1, estão dispostas as especificações técnicas do módulo PV utilizado. A partir dos dados apresentados, foi possível determinar a potência instalada para as duas UCs. Logo, o sistema fotovoltaico de cerca de 4,74 kWp de potência instalada pode atender a necessidade energética para a UC da cidade de Redenção, e para a UC em Maputo, a potência instalada seria 4,33 kWp.

QUADRO 1. Especificações técnicas do módulo FV.

\begin{tabular}{cc}
\hline Parâmetros & Valor \\
\hline Máxima Potência $(\mathrm{Pm})$ & $325 \mathrm{~W}$ \\
Tolerância: & $0 / 5 \mathrm{~W}$ \\
Tensão de Máxima Potência (Vm): & $37,6 \mathrm{~V}$ \\
Corrente de Máxima Potência (Im): & $8,64 \mathrm{~A}$ \\
Tensão de Circuito Aberto (Voc): & $46,0 \mathrm{~V}$ \\
Corrente de Curto-Circuito (Isc): & $9,24 \mathrm{~A}$ \\
\hline
\end{tabular}
Fonte: NEOSOLAR (2019).

Observa-se que potência instalada para a UC de Maputo foi menor do que a UC de Redenção, isso foi devido Maputo apresentar maiores índices de irradiação ao logo do ano em comparação aos dados de Redenção. Os procedimentos para determinação de cada componente do sistema fotovoltaico que foram apresentados na Figura 2 são: arranjo FV; Inversor e os demais elementos do sistema de potência.

\section{Arranjo FV}

Para o arranjo fotovoltaico utilizou-se os módulos da marca Bringing Green Power to Life cuja potência nominal de cada módulo é $325 \mathrm{KWp}$. Para atender à necessidade da UC da cidade de Maputo o sistema é composto por 14 módulos em dois strings conectados em série. Cada string (7 módulos) foi conectada a um inversor.

Porém, para a UC de Redenção, o sistema é composto por 16 módulos em dois strings conectados em série. Cada string (8 módulos) foi conectada a um inversor. Observa-se que para atender o mesmo consumo de $794 \mathrm{kWh}$, o sistema instalado em Maputo utilizaria menos módulos fotovoltaicos em comparação ao 
sistema da cidade de Redenção. Assim, verifica-se que o sistema em Maputo sairia mais barato financeiramente. Porém, a instalação do sistema fotovoltaico para as duas UCs é viável, visto nas duas cidades apresentam ótimas condições de irradiação ao logo do ano.

\section{Inversor}

O processamento e condicionamento da energia proveniente de um sistema PV foram realizados por um inversor. Para simulação, utilizou um conversor CC-CC e um conversor CC-CA. Na prática, poderia ser utilizado um inversor Grid-Tie Fronius Primo 4.0 que tem potência nominal de $4 \mathrm{kWp}$ na saída (ANEEL, 2016). Este inversor foi compatível para as instalações nas duas cidades, visto que, o FDI para a UC em Maputo é igual a 92,4\%, e em Redenção é $84,5 \%$. Nas duas situações o inversor foi compatível com o sistema proposto, atendendo a faixa da equação (7).

\section{Demais elementos do sistema de potência}

A indutância e a capacitância do filtro LC de saída foram especificadas. Já as capacitâncias de entrada dos conversores CC/CC e CC/CA foram determinadas.

\section{CONSIDERAÇÕES FINAIS}

A energia gerada a partir de dois sistemas fotovoltaicos das duas UCs localizados um na província de Maputo em Moçambique, e outro, na cidade de Redenção no Brasil, se verifica que na região de Maputo o sistema pode gerar mais energia elétrica em comparação à cidade de Redenção.

Dessa forma, define-se uma potência para os dois exemplos de projeto. Para primeiro exemplo uma potência de $4,33 \mathrm{kWp}$ pode atender a necessidade de Maputo. No caso do segundo exemplo, o sistema fotovoltaico de 4,74 kWp de potência instalada pode ser suficiente para o consumo médio mensal da cidade de Redenção.

Observa-se que a potência instalada para a UC de Maputo é menor do que a UC de Redenção, isso é devido Maputo apresentar maiores índices de irradiação ao logo do ano em comparação aos dados de Redenção. Assim, pode-se considerar que as duas regiões apresentaram dados favoráveis na geração de energia fotovoltaica.

\section{REFERÊNCIAS}

ABDALA, P. J. P. Energia solar e eólica. Editora Atena, Ponta Grossa, v.1, 2019, 432p. Disponível em: <https://www.atenaeditora.com.br/wpcontent/uploads/2019/01/E-book-Energia-Solar-e-E\%C3\%B3lica.pdf>.

ABSOLAR. Associação Brasileira de Energia Solar Fotovoltaica Geração Distribuída Solar Fotovoltaica. ENASE. Rio de Janeiro, 2016. Disponível em: $<$ http://www.absolar.org.br/>.

AIM. Relatório Energético da África Austral. Africa Inland Mission Internacional. 2012. Disponível em: <www.aimint.org>.

ANEEL. Resolução Normativa N 482/2012. 17/04/12 ed. 2012. Disponível em: <http://www2.aneel.gov.br/arquivos/PDF/Resolu\%C3\%A7\%C3\%A30\%20Normativa \%20482,\%20de\%202012\%20-\%20bip-junho-2012.pdf>. 
ANEEL. Resolução Normativa N 687/2015. 24/04/15. ed, 2015. Disponível em: <http://www.aneel.gov.br/cedoc/ren2015687.pdf>.

ANEEL, Procedimentos de Distribuição de Energia Elétrica no Sistema Elétrico Nacional. PRODIST, Módulo 3, Acesso ao Sistema de Distribuição. Rev. 7. 2016. Disponível em: <http://www.aneel.gov.br/documents/656827/14866914/PRODISTM\%C3\%B3dulo3_Revis\%C3\%A3o7/ebfa9546-09c2-4fe5-a5a2-ac8430cbca99>.

ANEEL. Nota Técnica $\mathbf{n}^{\circ}$ 0056/2017-SRD/ANEEL. 24/05/17 ed, 2017. Disponível em:<http://www.aneel.gov.br/documents/656827/15234696/Nota+T\%C3\%A9cnica_0 056_PROJE\%C3\%87\%C3\%95ES+GD+2017/38cad9ae-71f6-8788-0429d097409a0ba9>.

AYRES, B. F. Custo de disponibilidade e a energia solar fotovoltaica. 2019. Disponível em:<http://sharenergy.com.br/custo-de-disponibilidade-e-a-energia-solarfotovoltaica/>.

BORGES, T. H. Panorama da Geração Distribuída de Energia Elétrica no Brasil: Avanços e Desafios. Revista Especialize On-line, n.13, v.1, 2017. Disponível em: $<$ https://www.ipog.edu.br/download-arquivo-site.sp?arquivo=teddy-henrique-borges1886919.pdf>.

BRASIL. Ministério de Minas e Energia. Plano decimal de expansão de energia 2023. 2014, 433p. Disponível em: <http://www.mme.gov.br/documents/10584/1256600/PDE2023_ConsultaPublica.pdf/f 1cba0f0-1287-4d9a-9835-74f276980a43>.

BRASIL. Cartilha Energia: como analisar gastos com energia elétrica - Ministério do Planejamento, Orçamento e Gestão, Assessoria Especial para Modernização da Gestão, Central de Compras. Brasília, 2015, 24p. Disponível em: <http://www.mme.gov.br/documents/10584/1985241/Manual\%20de\%20Tarif\%20En \%20El\%20-\%20Procel_EPP\%20-\%20Agosto-2011.pdf>.

BRASIL. Ministério de Minas e Energia. Boletim mensal de monitoramento do setor elétrico - dezembro de 2016. Brasília: MME, 2017. Disponível em: <http://www.mme.gov.br/documents/10584/3308684/Boletim+de+Monitoramento+do +Sistema+El\%EF\%BF\%BDtrico+-+Dezembro-2016.pdf/f6b5284d-4105-4b79-a030$31755664721 \mathrm{a}>$.

CAMIOTO, F. C.; GOMES, V. P. R. G. análise de viabilidade econômica da implantação de um sistema de energia fotovoltaico nas residências uberabenses. Revista Produção Online, v. 18, n. 4, p. 1159-1180, 2018. Disponível em: <https://www.producaoonline.org.br/rpo/article/view/2649/1731>.

CARNEIRO, J. Dimensionamento de sistemas fotovoltaicos. 2009, 37p. Disponível em: <https://repositorium.sdum.uminho.pt/bitstream/1822/16965/1/DIMENSIONAMENTO \%20DE\%20SISTEMAS\%20FOTOVOLTAICOS.pdf>.

CEPEL - CRESESB. Manual de Engenharia para Sistemas Fotovoltaicos, p. 17, 
31, 43, 26/ Rio de Janeiro, Edição Especial, PRC-PRODEEM. Rio de Janeiro Agosto - 2004.

DANTAS; S. G.; POMPERMAYER, F. M. Viabilidade econômica de sistemas fotovoltaicos no Brasil e possíveis efeitos no setor elétrico. Instituto de Pesquisa Econômica Aplicada (IPEA), Rio de Janeiro, 2018, 42p. Disponível em: $<$ http://www.ipea.gov.br/portal/images/stories/PDFs/TDs/td_2388.pdf>.

EPE - Empresa de Pesquisa Energética. Nota Técnica DEA 19/14 - Inserção da Geração Fotovoltaica Distribuídos no Brasil - Condicionantes e Impactos. Rio de Janeiro, outubro, 2014.

EPE. Consumo Anual de Energia Elétrica por classe (nacional) - 1995-2016. 2017. Disponível em: <http://www.epe.gov.br/pt/publicacoes-dadosabertos/publicacoes/Consumo-Anual-de-Energia-Eletrica-por-classe-nacional>.

GTES - Grupo de Trabalho de Energia Solar, CEPEL - CRESESB. Manual de Engenharia para Sistemas Fotovoltaicos, Rio de Janeiro, agosto, 2008.

INEE - Instituto Nacional de Eficiência Energética. O que é Geração Distribuída. 2018. Disponível em: <http://www.inee.org.br/forum_ger_distrib.asp>.

INAM. Instituto Nacional de Meteorologia de Moçambique, 2018. Disponível em: $<$ http://www.sitesdemocambique.com/links-uteis/instituto-nacional-de-meteorologiamocambique/453>.

LEVA, F. F.; SALERNO, C. H.; CAMACHO, J. R.; GUIMARÃES, S. C. Modelo de um projeto de um sistema fotovoltaico, Encontro de Energia no Meio Rural, v. 5, 2004. Disponível em: <http://www.proceedings.scielo.br/scielo.php?pid=MSC0000000022004000200020\& script=sci_arttext>.

NASCIMENTO, R. L. Energia solar no Brasil: situação e perspectivas. Estudo Técnico, 2017, 46p. Disponível em: $<$ http://bd.camara.gov.br/bd/bitstream/handle/bdcamara/32259/energia_solar_limp.p df? sequence $=1>$.

NEOSOLAR. Bringing Green Power to Life. Painel Solar Fotovoltaico GCL-P6/72 330Wp (Datasheet). 2019. Disponível em: <https://www.neosolar.com.br/loja/painelsolar-fotovoltaico-gcl-p6-72-330wp.html>.

PEREIRA, E. B; MARTINS, F.R.; ABREU, S. L.; RÜTHER, R. Atlas Brasileiro de Energia Solar. INPE, 2006, 64p. Disponível em: $<$ http://ftp.cptec.inpe.br/labren/publ/livros/brazil_solar_atlas_R1.pdf>

PINHO, J. T.; GOLDINO, M. A. Manual de engenharia para sistemas fotovoltaicos. 2014, 530p. Disponível em: <https://www.portalenergia.com/downloads/livro-manual-de-engenharia-sistemas-fotovoltaicos2014.pdf>. 
SANTOS, M. H. S.; VIANA, T. S.; SOUZA, M. C. L.; AVELINO, M. R.; WEBER SILVA, W. S. Sistemas fotovoltaicos conectados à rede para Moçambique. Acta Scientiae \& Technicae. v. 1, n. 2. 2013. Disponível em: <http://www.uezo.rj.gov.br/ojs/index.php/ast/article/view/36>. doi: https://doi.org/10.17648/uezo-ast-v1i2.36.

SOUZA, A.C.; SILVA, L. R. C.; OLIVEIRA, T. L.; J. R.; MACEDO JR, J. R.; SANTOS, I.N. Análise da microgeração fotovoltaica monofásica na rede de distribuição de baixa tensão. CEEL, p.1-6, 2016. Disponível em: $<$ https://www.peteletricaufu.com/static/ceel/doc/artigos/artigos2016/ceel2016_artigo1 68_r01.pdf>.

TAB ENERGIA. Energia solar: Brasil e Alemanha, como reverter o 7X1, 2019. Disponível em: <https://www.tabenergia.com.br/post/energia-solar-brasil-ealemanha-como-reverter-0-7x1>.

WEATHER SPARK - Condições meteorológicas médias de Redenção, 2019. Disponível em: <https://pt.weatherspark.com/y/31109/Clima-caracter\%C3\%ADsticoem-Reden\%C3\%A7\%C3\%A3o-Brasil-durante-o-ano>.

ZILLES, R.; MACEDO, W.N.; GALHARDO, M.A.B.; OLIVEIRA, S.H.F.;Sistemas fotovoltaicos conectados à rede elétrica. $1^{\underline{a}}$ ed. São Paulo: Oficina de Textos, 2012. 